OPEN ACCESS

Edited by:

Dariusz Leszczynski,

University of Helsinki, Finland

Reviewed by:

David Welch,

Columbia University, United States

Kasper Grandahl,

Holbæk Sygehus, Denmark

*Correspondence:

Nathan J. Downs

downsn@usq.edu.au

Specialty section:

This article was submitted to

Radiation and Health,

a section of the journal

Frontiers in Public Health

Received: 14 July 2017 Accepted: 03 November 2017 Published: 16 November 2017

Citation:

Downs NJ, Parisi AV, Butler H, Rawlings A and Elrahoumi RS (2017) An Inexpensive High-Temporal

Resolution Electronic Sun

Journal for Monitoring Personal Day

to Day Sun Exposure Patterns.

Front. Public Health 5:310.

doi: 10.3389/fpubh.2017.00310

\section{An Inexpensive High-Temporal Resolution Electronic Sun Journal for Monitoring Personal Day to Day Sun Exposure Patterns}

\author{
Nathan J. Downs*, Alfio V. Parisi, Harry Butler, Alex Rawlings and Raja Salem Elrahoumi \\ Faculty of Health, Engineering and Sciences, University of Southern Queensland, Toowoomba, QLD, Australia
}

Exposure to natural sunlight, specifically solar ultraviolet (UV) radiation contributes to lifetime risks of skin cancer, eye disease, and diseases associated with vitamin $D$ insufficiency. Improved knowledge of personal sun exposure patterns can inform public health policy; and help target high-risk population groups. Subsequently, an extensive number of studies have been conducted to measure personal solar UV exposure in a variety of settings. Many of these studies, however, use digital or paper-based journals (self-reported volunteer recall), or employ cost prohibitive electronic UV dosimeters (that limit the size of sample populations), to estimate periods of exposure. A cost effective personal electronic sun journal (ESJ) built from readily available infrared photodiodes is presented in this research. The ESJ can be used to complement traditional UV dosimeters that measure total biologically effective exposure by providing a time-stamped sun exposure record. The ESJ can be easily attached to clothing and data logged to personal devices (including fitness monitors or smartphones). The ESJ improves upon self-reported exposure recording and is a cost effective high-temporal resolution option for monitoring personal sun exposure behavior in large population studies.

Keywords: sun journal, ultraviolet, infrared, erythema, skin cancer, vitamin D

\section{INTRODUCTION}

A number of devices and innovative methodologies have recently been promoted advocating the benefits of monitoring personal exposure patterns to solar radiation. These innovations are beneficial to the community and researchers as tools for monitoring sun-going exposure behavior. The quality of commercially available sun monitoring devices depends to some extent on the costs associated with the respective technologies implemented in their design. An inexpensive design is discussed in this research as an alternative for many in the research community utilizing self-reporting of personal sun exposure behavior as a method for studying exposure in study participants. The aim of these studies is often to effect a reduction in the disease burden related to the natural exposure of the skin and eye to sunlight. This is a significant public health issue as the terrestrial ultraviolet spectrum (UV, 290-400 nm) is strongly associated with the development of keratinocyte skin cancers (1-3), eye disease (4), and malignant melanoma (5) - the most aggressive form of skin cancer in humans. The exposure received by an individual venturing into an outdoor environment is dependent upon the available ambient UV at the time of exposure. The ambient UV is dependent on the solar zenith 
angle (SZA) and other physical factors including cloud cover (6), aerosol concentration, and total column ozone $(7,8)$.

Irrespective of the available ambient UV, outdoor exposure behavior, and attitudes to prevention (including risk minimization through personal protection) are the most significant factors influencing personal UV dose $(9,10)$. How an individual interacts within an outdoor environment, and the timing of any outdoor exposure will significantly modify personal cumulative UV exposure, and the acute intermittent doses that lead to episodes of sunburn. Much work has therefore been done in studying outdoor behavior patterns and encouraging preventative risk strategies for different populations, including occupational and sporting groups (11-15).

To quantitatively assess the personal sun exposure of individuals, cumulative UV exposure is typically measured using polysulphone (16) or biological spore films (17), while behavior patterns are monitored (often by trained study participants) with the use of sun journals $(14,18)$. Once calibrated, polysulphone and spore-type dosimeters (low-cost) can provide single use measurements of personal exposures up to a maximum saturation limit. For polysulphone dosimeters, measureable exposure periods typically do not extend beyond 1 day in tropical climates (19). Electronic UV dosimeters (high-cost) have been developed and utilized in several studies to assess personal outdoor exposure patterns to a finer level of detail (often measured in second-long intervals) than is possible using paper-based sun journals (9, 20, 21). Electronic UV dosimeters have many advantages over cumulative film-type badges, including unrestricted exposure limits, reusability, and the elimination of recall error by study participants (22). A significant disadvantage is the associated purchase cost of UV sensitive photodiodes. Consequently, these costs limit the total number of electronic dosimeters available for detailed personal exposure studies and therefore the sample size of those studies. Hence, sun journals are still favored where the study requires a large sample size to infer outdoor exposure behavior for large populations.

Effective UV radiation dosimetry and personal sun exposure monitoring through the use of sun journals is often a compromise between study population size and cost. When initially developed, the electronic UV dosimeter was shown to be an effective personal UV monitor, capable of providing high-temporal resolution exposure information (23). The high cost of earlier electronic UV dosimeters was offset somewhat by the reusability of the design. Fortunately, since pre-21st century electronic UV dosimetry was introduced, costs for Silicon Carbide $(\mathrm{SiC})$ and Aluminum/Indium (Al/In) Gallium Nitride (GaN) UV photodiodes have become more affordable. These specialized photodiodes have resulted in an approximate 10 -fold reduction in cost per dosimeter unit when compared with earlier designs. Recently, SiC and (Al/In) GaN photodiodes have been amalgamated successfully with wrist bands and lapel badges, providing high-temporal resolution time-stamped personal UV exposures $(22,24-27)$. These electronic dosimeters are sensitive to biologically effective short wavelength UVB radiation $(290-315 \mathrm{~nm})$. However, they suffer from low-signal outputs (in the order of $\mathrm{nA}$ ) and hence require amplification to achieve readings in the $0-2.5 \mathrm{~V}$ range $(26,28)$. Signal amplification adds an additional cost to the required electronics of the UV dosimeter, consisting also of a data logger and control electronics. Like spore and film-type dosimeters, electronic UV dosimeters are typically calibrated to the erythemal action spectrum (29) to derive the integral of time-stamped solar irradiance data as a biologically effective exposure in units of joule per square meter or standard erythema dose (30). Erythemal solar UV exposures recorded by electronic UV dosimeters may also be expressed in terms of the UV index (31).

The high level of precision associated with accurate electronic UV dosimeters have recently been supplemented with alternative commercial methods of monitoring personal solar exposure, including lower cost electronic UV bracelets linked to smartphones (32), badges employing photosensitive dyes (33, $34)$, as well as beads and bangles sensitive to solar UV radiation $(35,36)$. While many commercial options appear to provide cost effective solutions for monitoring behavior, they often provide limited modes of operation and lack the design flexibility necessary for researchers interested in studying personal sun exposure patterns. Table 1 summarizes a variety of personal sun exposure monitoring devices currently available to the public and research community for personal solar radiation monitoring. Costs are expressed per unit and approximated in US dollars (\$US).

The measurement of changing exposure, due to variations in: (i) the available ambient UV and (ii) changes in personal exposure behavior are of significant interest to researchers who need to quantify personal exposure in different populations. While paper-based sun journals exist as cost effective alternatives to electronic UV dosimeters they are not effective tools for recording personal exposure patterns at high resolutions, and may be prone to recall error by study participants. An electronic sun journal (ESJ) utilizing an inexpensive infrared photodiode has been developed in this research to log personal outdoor exposure time at high-sampling rates. The introduction of an infrared photodiode for the ESJ, utilizes existing photodiode technology commonly employed for remote control and door sensor systems. Typical purchase costs for individual infrared photodiodes range from $<\$ 1$ to $\$ 5$ US. Thus, the ESJ introduces a novel methodology for sensing outdoor exposure patterns not previously tested for personal solar exposure monitoring. This technology offers a substantial cost saving over existing UV photodiodes where the ESJ also replaces the traditional paper-based sun journal while retaining the advantages of a high-electronic sampling rate. The developed ESJ uses an infrared photodiode that is optimized to detect periods of outdoor exposure, given the abundance of naturally occurring infrared radiation in sunlight and its relative absence in indoor locations. When connected to a suitable data logger (smartphone or portable logger), the ESJ can accurately monitor personal outdoor behavior patterns.

\section{MATERIALS AND METHODS}

An infrared photodiode [BPV22F, Vishay Semiconductors (37)] was tested for use as the primary input sensor of the ESJ. The BPV22F is designed by the manufacturer for high-radiant 
TABLE 1 | Cost and function of methods available for monitoring personal exposure to solar radiation.

\begin{tabular}{|c|c|c|c|c|c|}
\hline Function & UV sensitive component & Approximate cost & Typical exposure & Reusable & Flexible sample rate \\
\hline \multicolumn{6}{|l|}{ UV dosimeter } \\
\hline Polysulphone/spore & Sensitive polymer/biological & $\$ 1-\$ 10$ & 1 day & No & No \\
\hline Electronic & SiC photodiode & $\$ 220^{a, b}$ & 1 week & Yes & Yes \\
\hline Electronic & (Al/In)GaN photodiode & $\$ 40^{\mathrm{b}, \mathrm{c}}$ & 1 week & Yes & Yes \\
\hline \multicolumn{6}{|l|}{ Infrared sun journal $^{d}$} \\
\hline Electronic & Infrared photodiode & $\$ 1^{\mathrm{b}, \mathrm{d}}$ & 1 day & Yes & Yes \\
\hline \multicolumn{6}{|l|}{ Personal awareness } \\
\hline Bracelet & Sensitive polymer/dye & $\$ 7$ & 1 day & Yes & No \\
\hline Beads & Sensitive polymer/dye & $\$ 1$ & 1 day & Yes & No \\
\hline Cyanotype cloth & Sensitive dye & $\$ 3$ & $1 \mathrm{~h}$ & No & No \\
\hline \multicolumn{6}{|c|}{ Commercial UV monitor } \\
\hline Smartphone patches & Sensitive dye/image processing & $\$ 30$ & 1 week & No & No \\
\hline Smartphone bracelets & Light sensor & $\$ 100$ & 1 month & Yes & No \\
\hline
\end{tabular}

aPrice estimate is an average of commercially available SiC photodiodes sensitive to erythemal ultraviolet (UV).

${ }^{b}$ Cost does not include additional expense of data loggers, current amplifiers, or control electronics.

cPrice estimate is an average of commercially available AlGaN photodiodes sensitive to UVB.

${ }^{d}$ Proposed in this research. Average of 25 commercially available infrared photodiodes.

sensitivity over a wide range of receiving angles, is small and lightweight measuring $4.5 \mathrm{~mm} \times 5 \mathrm{~mm} \times 6 \mathrm{~mm}$, and has a hemispherical radiant sensitive area of $7.5 \mathrm{~mm}^{2}$. The photodiode is sensitive to the infrared-A waveband (870-1,050 nm, peak response $950 \mathrm{~nm}$ ). When operated in a reverse bias state and exposed to sunlight the diode response saturates. Conversely, in densely shaded environments or under indoor fluorescent lighting, which present a limited infrared source, the diode response is minimized.

The photodiode, when worn on an exposed part of the body such as the wrist or lapel, was tested for recording periods of indoor and outdoor exposure time. Personal exposure tests employing the BPV22F were conducted during the austral spring and summer months of 2016 and 2017. These tests were chosen to determine if the ESJ could accurately classify the difference between indoor and outdoor locations and included a shade response (sky view) test, static and personal outdoor environment tests, and an indoor incandescent light source test.

\section{Circuit Cost}

The total costs for the proposed ESJ are listed in Table 2. The circuit utilizes a TK-4703 voltage data logger [Gemini Data Loggers, UK (38)]. This is a commercially available data logger that was used in this research as it required no external assembly and included data download and control software (Tiny Tag Explorer ver 4.9, Gemini Data Loggers, UK). The circuit as designed will output a signal voltage between 0 and $2.5 \mathrm{~V}$ irrespective of the data logging unit connected at A (Figure 1). The design is therefore compatible with alternative data logging units, including smartphone memory, miniaturized computers, and SD card systems. These alternative data logger units are more affordable than many commercial voltage data loggers but may require further assembly depending on the logger design. Alternative purpose-built data loggers require at a minimum an analog to digital converter to covert a small $0-2.5 \mathrm{~V}$ signal to an eight-bit digital value that can be stored on SD card,
TABLE 2 | Approximate component costs for the electronic sun journal in \$US.

\begin{tabular}{lc}
\hline Component & Approximate cost (\$US) \\
\hline BPV22F infrared photodiode & $\$ 1.24$ \\
$2 \times 10 \mathrm{k} \Omega$ resistors & $2 \times \$ 0.34$ \\
$3 \vee$ coin cell battery & $\$ 1.41$ \\
$3 \vee$ battery holder & $\$ 0.92$ \\
TK-4703 data logger & $\$ 213.00$
\end{tabular}

${ }^{a}$ Alternative data loggers include micro SD card loggers ( $\$ 50$ US) or purpose-built microcontrollers.
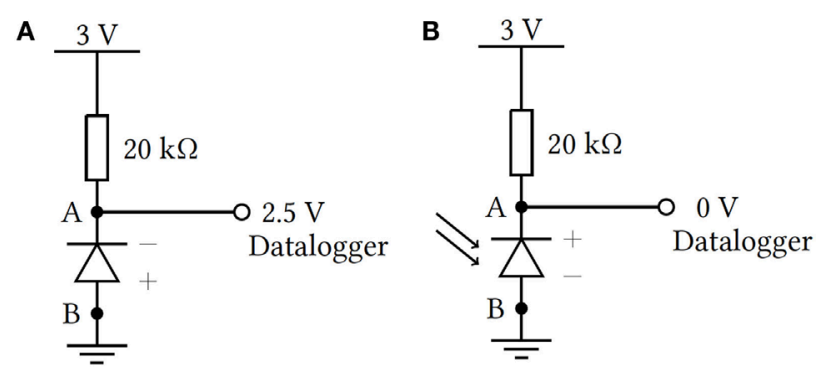

FIGURE 1 | Circuit design and operation under limited (A) and saturated (B) exposure states. Maximum output current occurs when the diode saturates and is limited to less than $0.1 \mathrm{~mA}$ to prolong battery life [(A) cathode, (B) anode].

EPROM, or alternative portable memory system such as a USB memory stick. Small microcontroller units designed to sample the ESJ voltage at user specified intervals are therefore capable of greatly extending the storage range of the device depending on the number of bytes of data storage available. Many DIY data logging kits and designs are made freely available on electronics forums accessible from the internet and would make very reasonable substitutes for commercial data loggers. Excluding the data logger, the complete ESJ circuit can fit within the circumference of a $3 \mathrm{~V}$ coin cell battery (measuring 
$20 \mathrm{~mm}$ in diameter). The design of a small transducer circuit could be optionally included to send monitored voltage signals digitally to offsite storage systems through the use of Wi-Fi to nearby computer or personal data storage devices including smartphones or portable tablet systems. The design presented here, however, requires minimal assembly, balancing the cost effectiveness of the infrared diode with an easy to use data logging unit for immediate operation.

\section{Circuit Design and Operation}

The ESJ circuit was designed for cost effectiveness and design flexibility. All listed components are interchangeable and based primarily on a simple voltage divider consisting of an infrared photodiode and resistor. For testing, the infrared photodiode (BPV22F) was connected in series with a $20 \mathrm{k} \Omega$ resistor and powered using a $3 \mathrm{~V}$ coin cell battery. The photodiode was connected to operate in the reverse bias condition with output voltage monitored in 1-s intervals using the TK-4703 voltage data logger. The TK-4703 has a memory capacity of 16 kilobytes and in this configuration could log exposures for $4 \frac{1}{2} \mathrm{~h}$. As designed, the total monitoring period is dependent on the flexible sampling rate of the ESJ and the available memory of the data logger unit. ESJ output can be monitored at very high-temporal resolutions or it can be lowered for studies that require monitoring over longer periods. The circuit design is shown in Figure 1A for limited exposure to sunlight (indoor condition) and Figure 1B for saturated (sunlight) conditions.

The photodiode circuit monitors the saturation state of the infrared diode within the limits of $0-2.5 \mathrm{~V}$. Under limited exposure conditions (indoors), the reverse bias of the photodiode restricts the flow of current from $A$ to $B$ (Figure 1A). In this state, the voltage divider including the $20 \mathrm{k} \Omega$ resistor is designed so that the voltage monitored at A reaches $2.5 \mathrm{~V}$. This maximum voltage is the highest voltage able to be recorded by the TK-4703 data logger.

When the sun saturates the photodiode, the $p-n$ junction semiconductor switches polarity, driving the cathode positive, and conducting from A to B (Figure 1B). In this condition (full sunlight), the circuit potential at $\mathrm{A}$ is $0 \mathrm{~V}$. Thus, the diode output ranges from $2.5 \mathrm{~V}$ for indoor conditions (low light), and falls to $0 \mathrm{~V}$ when exposed to full sunlight. Environments with limited infrared radiation, such as partially shaded locations, fall within the $0-2.5 \mathrm{~V}$ range. Thus, the circuit as designed is capable of monitoring the time spent in three states: (1) indoor conditions, (2) full sunlit conditions, and (3) semi-shaded environments.

\section{Cosine Response and Temperature Stability}

For the ESJ design presented here, the receiving infrared photodiode required a good cosine response to ensure personal measurements reflect the ambient exposure conditions. Technical specifications for the BPV22F indicate that the diode response matches the natural cosine function from $0^{\circ}$ to $80^{\circ}$ (37). The photodiode when worn on the arm, wrist, or lapel provides a recordable response for monitoring the times of indoor, full sunlight, and semi-shaded sunshine states of the wearer. A diffuser was not fitted to the BPV22F photodiode for this study. For designs utilizing different infrared diodes, the use of a diffuser to approximate the natural cosine response is recommended.

The circuit response was tested under temperature controlled laboratory conditions to ensure consistency in signal output during possible winter verses summertime use of the ESJ. To determine this response, the ESJ was placed within $10 \mathrm{~cm}$ of a glass-filtered infrared heating lamp. The glass filter reduced the circuit response to below the maximum output of 2.5 to $2.3 \mathrm{~V}$. The reduction in signal voltage ensured either a positive or negative change in output voltage could be monitored with increasing temperature. The output voltage at A (Figure 1) was monitored using an oscilloscope as the circuit was heated by a hair dryer unit from 16 to $60^{\circ} \mathrm{C}$. The temperature coefficient of the circuit monitored under these conditions was measured to be $-1.1 \mathrm{mV}^{\circ} \mathrm{C}^{-1}$. The measured ambient air temperature response of the BPV22F deployed in the ESJ circuit was better over this temperature range than the temperature coefficient stated by the manufacturer of $-2.6 \mathrm{mV}^{\circ} \mathrm{C}^{-1}$. The manufacturer of the BPV22F lists an operating range of the photodiode of between -40 and $100^{\circ} \mathrm{C}$. The reduction in measured signal voltage with expected changes in ambient air temperature is negligible compared with the full signal range of the ESJ ranging from 0-2.5 V.

\section{Sky View Test}

The BPV22F response saturates in natural sunlight where direct (un-shaded) solar radiation reaches the photosensitive area of the diode. Therefore, a sky view test was performed to determine the photodiode sensitivity to shaded outdoor exposure conditions. An open-air sky view test was completed in late spring (November $16,2016)$. For this test, a shadow shield $(113 \mathrm{~mm} \times 113 \mathrm{~mm})$ attached to a vertically upright stand assembly was moved from 0 to $885 \mathrm{~mm}$ above the surface of the diode during cloud-free midday conditions. Here, sky view was approximated according to the equation,

$$
S_{\%}=\frac{100}{2 \pi}[2 \pi-2 \pi(1-\sin \theta)],
$$

where the percentage sky view, $S_{\%}$ assumes a hemispherical field for the diffuse skylight of nominal radius 1 which is shaded by a square shadow of side length, $2 r_{\theta}$ as shown in Figure 2. The variable $r_{\theta}$ is a function of the shade shield height $(h)$ and may be calculated using:

$$
r_{\theta}=\cos \left(90-\tan ^{-1}\left(\frac{56.5}{h}\right)\right),
$$

where $h$, the height of the shield, is also illustrated in Figure 2. In the approximation of $S_{\%}$, the sky view covered by the shield shadow prevents radiation from the upper hemisphere as indicated in Figure 2 (red area) from reaching the diode. In all calculations of sky view, the size of the shadow is dependent upon the height of the shield, $h$ (in millimeters) above the photosensitive surface of the infrared diode. Here, the radius of the hemispherical sky view is always taken to be 1 irrespective of shield height. The maximum 
A

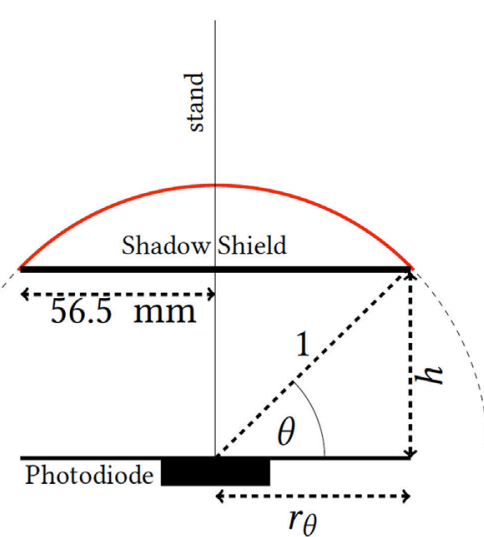

B

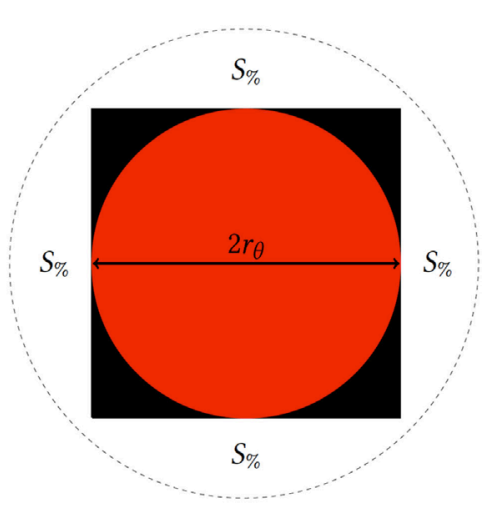

FIGURE 2 | Sky view assembly [(A) side and (B) top view] used to test the infrared photodiode sensitivity to increasing levels of diffuse solar radiation (red-shaded sky hemisphere, black - shade shield, dotted circle-visible sky view of nominal radius 1).

length of $r_{\theta}$ will be 1 when the shield is $0 \mathrm{~mm}$ above the diode surface and will reduce in proportion to the visible sky view as $h$ increases.

The tested height range for the shadow shield equated to an approximate range in shaded sky view of 0-99.8\% measured over 20 steps (approximately 5\% increments in sky view). During each step, the shadow cast by the shield attached to the stand assembly was moved such that the diode remained near the center as illustrated in Figure 2. All height adjustments of the shadow shield were performed within $1 \mathrm{~h}$. This ensured minimal movement of the solar disc for the duration of each step interval.

\section{Indoor Incandescent Response Test}

The ESJ was tested under an incandescent light source to determine the distance at which the ESJ would incorrectly record an outdoor exposure state. Here, the personally monitored indoor condition of the ESJ assumes little or no infrared radiation reaches the sensitive surface of the photodiode to produce a $2.5 \mathrm{~V}$ signal output in the reverse bias state. This is true for most indoor lighting conditions where artificial fluorescent or LED lighting is used. This is also true for energy efficient low-power light globes which produce a discrete spectrum. However, where older incandescent indoor lighting is used (continuous spectrum lighting) the distance from the light source (where the source intensity varies proportionally with the square of the distance) will be a contributing factor. The sensitivity of the ESJ was tested by noting the incandescent globe response in situ by placing the BPV22F infrared diode from between 0.1 and $1 \mathrm{~m}$ from a $100 \mathrm{~W}$ incandescent light globe. Exposure response was measured in $10 \mathrm{~cm}$ steps. For this test, the light globe was fixed to a shielded and blackened box assembly to ensure only infrared radiation from the source was measured. The shielded box measured $0.9 \mathrm{~m} \times 0.6 \mathrm{~m} \times 0.6 \mathrm{~m}$.

\section{Field Trials and Static Response Test}

The ESJ photodiode signal output was tested under actual outdoor shade conditions to determine if a difference could be found between a static shade structure, and tree canopies that provided both heavy and light shade protection. Each of these static response tests were conducted over $10 \mathrm{~min}$ duration by placing the ESJ on the ground within the approximate middle of the defined shaded region for each environment.

The response of the ESJ was also studied for an individual walking between and through different outdoor environments offering varying levels of shade protection to determine if the ESJ could accurately classify periods of indoor exposure, periods within shaded environments, and periods located in direct sunlight. A total of nine personal field tests were conducted between September 2016 and March 2017. Trials were held at various times of day for periods ranging from 1 to $31 / 2 \mathrm{~h}$ under different ambient conditions including clear sky and cloud-affected periods. All trials were conducted at the University of Southern Queensland Toowoomba campus $\left(27.5^{\circ} \mathrm{S}, 152^{\circ} \mathrm{E}\right)$, and involved volunteers walking the ESJ held and maintained in a horizontal plane in the palm of the hand to and from at least three separate locations on campus. These locations included buildings (indoors), between building structures, heavily and lightly shaded environments protected by trees, various shade structures, and open outdoor environments.

\section{RESULTS}

\section{Sky View Test}

Figure 3 gives the output voltage of the ESJ relative to the sky view. As the shaded sky view approaches $100 \%$ the output voltage falls quickly to $0 \mathrm{~V}$. Any exposure to direct (un-shaded) solar radiation will saturate the photodiode also resulting in a measurement of $0 \mathrm{~V}$. Therefore, any monitored readings of $0 \mathrm{~V}$ are due to either exposure to solar radiation in an open environment that approaches near $100 \%$ sky view or an exposure to direct solar radiation. The outdoor response of the ESJ as designed is not sensitive to diurnal variation in radiation intensity (which occurs with changing SZA) and will saturate even under cloudy conditions. Response measurements greater than $0 \mathrm{~V}$ are due to the ESJ being exposed to an environment that provides a level 


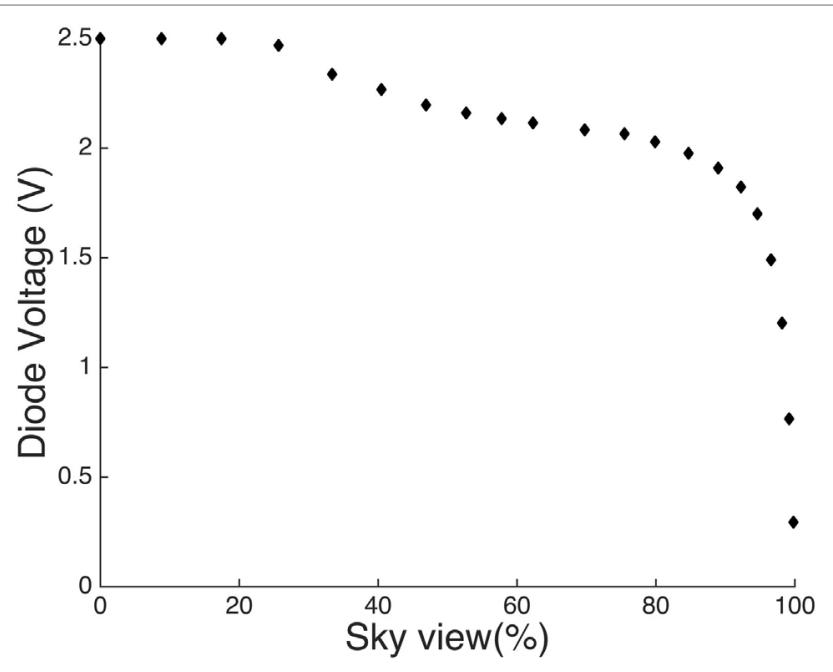

FIGURE 3 | Photodiode voltage plotted as a function of sky view.

of protection from both direct and diffuse radiation (i.e., shaded environments).

\section{Incandescent Globe Response Test}

As seen in Figure 4, the response of the BPV22F is not significant until the photodiode is placed within $0.5 \mathrm{~m}$ of the $100 \mathrm{~W}$ incandescent globe. Used as a personal sunshine monitor, the designed circuit will therefore detect indoor conditions $(2.5 \mathrm{~V})$ for most instances, provided wearers are not located within close proximity to an incandescent light source. Similarly for bar heaters or radiators, wearers would need to be located in close proximity to the source to receive a false outdoor reading. Given the position of most indoor lighting sources being fixed to the ceilings of buildings, the developed ESJ is not expected to record false outdoor states when wearers are indoors.

\section{Monitoring the Environment Response}

The ESJ is sensitive to shade in the outdoor environment. The response voltage for three static environments on the Toowoomba USQ campus is shown in Figure 5. Characteristic of each outdoor environment is the increased output voltage of the ESJ with increasing shade density (reduced sky view). Also noticeable in Figure 5 is the influence of shade provided by a fixed shade structure compared with trees and natural vegetation. Fixed structures, such as verandahs, awnings, and purpose-built shade covers provide a consistent level of shade protection at a constant SZA resulting in a constant ESJ output as evident in Figure 5 (upper dark line). For trees and natural vegetation, movement of canopy leaves and branches produces noticeable fluctuations in the output response voltage. For the tree that provided the least level of shade protection, the changing orientation of the shade pattern with respect to the movement of the solar disc (changing SZA) is evident by the steady increase in the monitored output voltage (bottom red line in Figure 5). The qualitative level of shade protection can be approximated by the ESJ voltage for an individual monitored in a stationary condition under the shade of a tree or static structure.

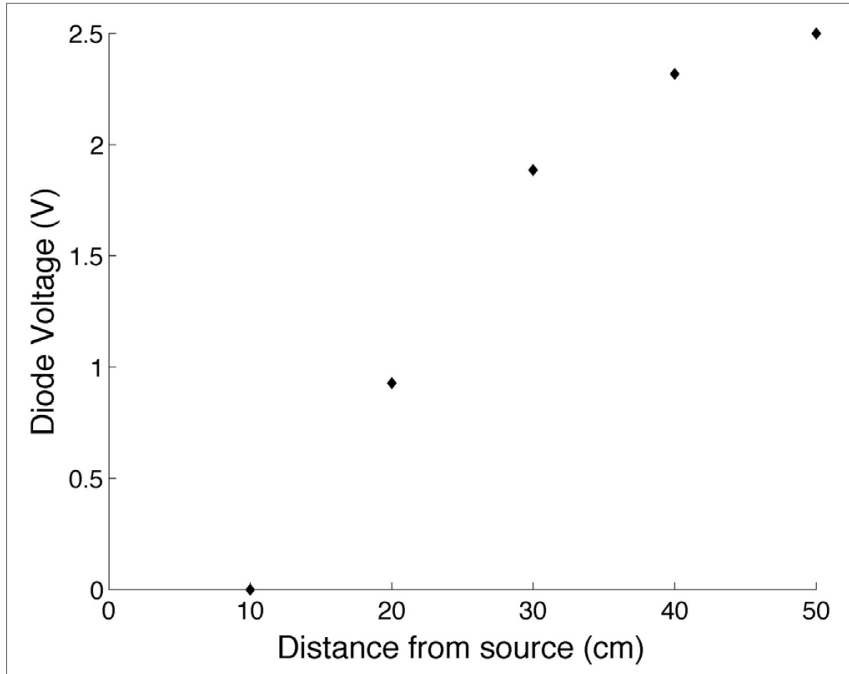

FIGURE 4 | Photodiode voltage versus distance from an incandescent light globe.

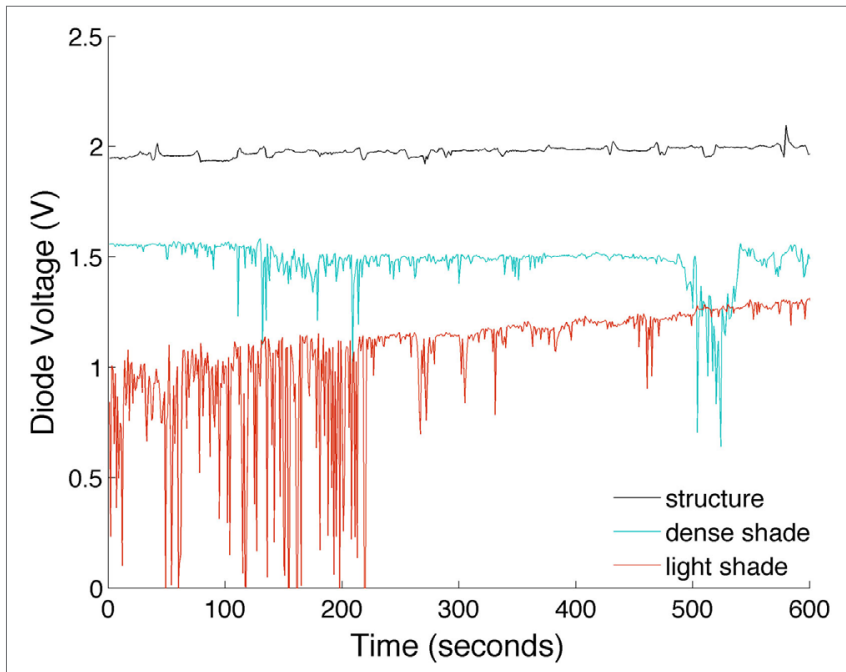

FIGURE 5 | Electronic sun journal output monitored in different static outdoor environments (upper line-shade structure, middle blue-dense tree shade, bottom red-light tree shade).

\section{Field Trials}

The voltage output of the infrared ESJ is provided for each of the nine field trials conducted from September 2016 to March 2017 in Figure 6. Visible in each trial frame of Figure $\mathbf{6}$ are periods of exposure to open and shaded outdoor environments. Indoor environments show the saturated condition of the ESJ at $2.5 \mathrm{~V}$. To highlight personal exposure patterns, periods of outdoor exposure $(0 \mathrm{~V})$ are colored red, shaded outdoor exposures are uncolored $(0-2.5 \mathrm{~V})$, and indoor exposure periods $(2.5 \mathrm{~V})$ are colored green. When worn on an exposed surface of the body, such as the wrist, the ESJ can provide quantitative information of an individual's day to day outdoor exposure pattern.

The circuit as designed is capable of detecting three personal sunshine conditions, (i) indoor environments; (ii) shaded 
A

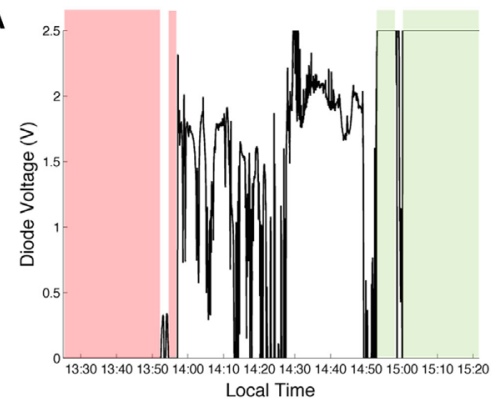

D

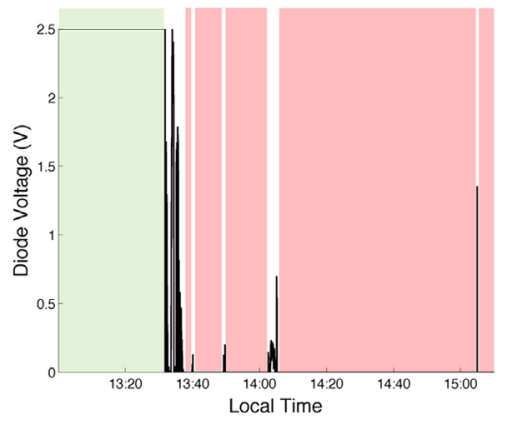

G

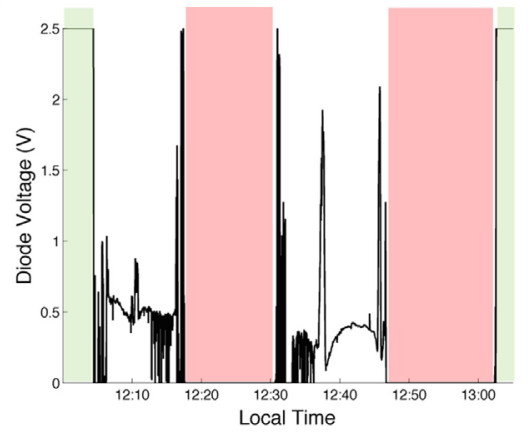

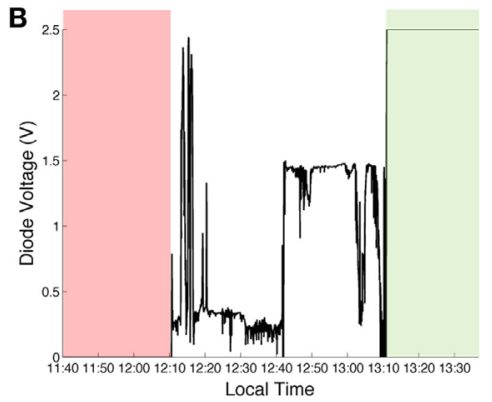

E

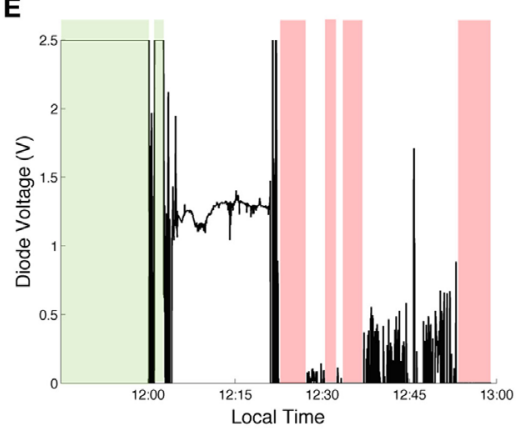

H

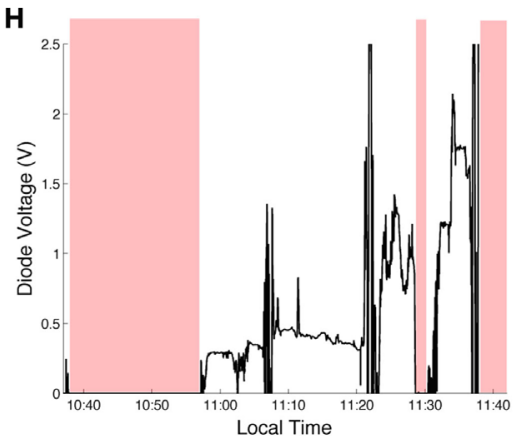

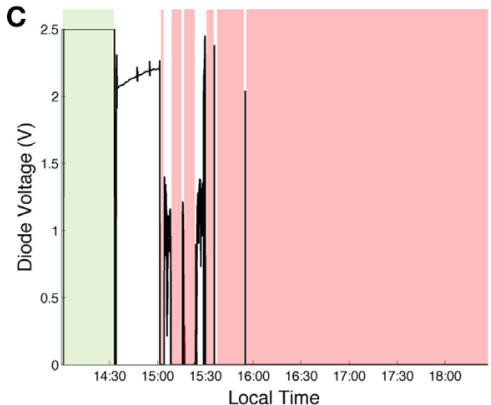
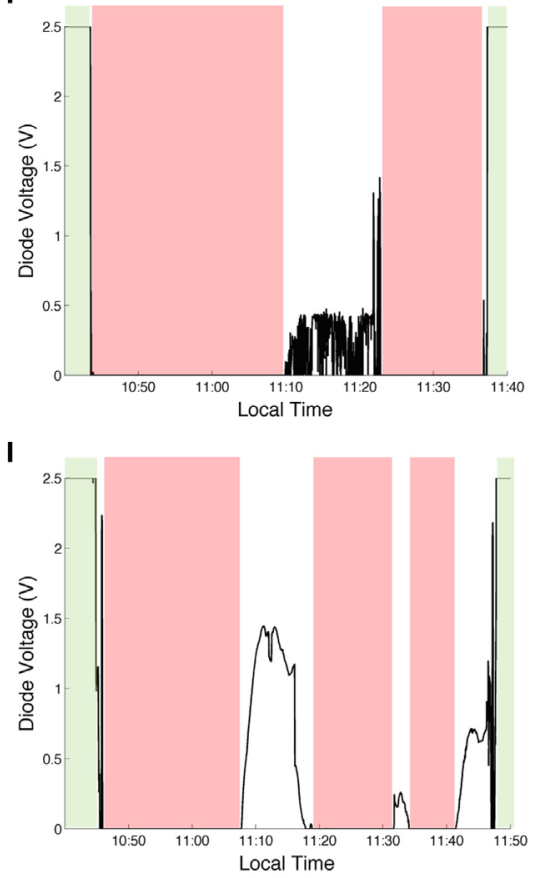

FIGURE 6 | Personal outdoor exposure pattern determined from the voltage output of the electronic sun journal for an individual moving about an outdoor environment (A) 22 September; (B) 24 September midmorning, (C) 24 September afternoon, (D) 26 September; (E) 27 September; (F) 18 January midmorning; (G) 18 January afternoon; (H) 1 February; and (I) 29 March.

environments, and (iii) open outdoor environments. As shown in Figure 6, the diode output reaches its maximum condition only in well-protected indoor environments in the absence of an infrared radiation source. This condition is made obvious by the static maximum output noted by the data logger at $2.5 \mathrm{~V}$ (green highlighted regions). Exposure to the near infrared causes an immediate diode response and drop in voltage. In this research, we classify semi-shaded environments as those in which a fluctuating voltage is measured between 0 and $2.5 \mathrm{~V}$. The fluctuation in voltage can be used to assess the quality of shade provided as an individual moves about the environment. Figure 6B, for example, provides a good indication that the participant moved from one outdoor environment that provided some level of protection from the sun to an outdoor environment that provided a better level of protection as shown between 12:10 and 13:10 p.m. by the increase in voltage signal. The lower signal variability indicated in the Figure 6B frame suggests these environments were likely to be static shade structures. Comparing this output to the shaded condition of Figure $6 \mathrm{~F}$ as another example, hints at the likelihood that the wearer may have been placed under a tree canopy as the signal varied rapidly between 11:00 and 11:20 a.m. Thus, the ESJ provides information on personal shade seeking habits of individual wearers and the type of environments they visit when outdoors. This is useful information that could be used to examine the shade seeking behavior of individuals and study participants.

\section{DISCUSSION}

The presented technique employs the use of readily available and inexpensive infrared photodiodes for use as an ESJ. When employed for use as a practical cost effective high-temporal resolution sun journal, the infrared photodiode provides sufficient information on the ambient environment experienced by the wearer to log details on behavioral exposure patterns, not able to be recorded to such high resolution using traditional self-reported sun diaries. Highsampling rates improve the accuracy of long-term epidemiological 
cumulative exposure studies. They also enable precise measurement of intermittent or incidental sun exposure patterns while removing the burden placed on study participants to recall past sun exposure behavior. High-temporal resolution exposure pattern data may be beneficial to studies that seek to determine shade use in school playgrounds, public spaces, or the working environment. It may also be useful for determining exposure patterns in different professions. Being a cost effective alternative to existing electronic UV dosimeters, the ESJ could also be utilized in larger study populations.

The ESJ is not a UV dosimeter. However, when the ESJ is combined with traditional spore or film-type badges, it may be used to provide time-stamped information on personal exposure habits. This information can be conveyed by the ESJ more reliably than by study participants using paper-based sun journals. Sun exposure pattern information, including periods of indoor, outdoor, and partially shaded exposures would aid UV dosimeter studies seeking to determine cause and effect relationships in different population groups.

The ESJ as designed and tested in this research is not waterproof. Rather our results focus on the development of a novel approach and circuit design that can be adopted by manufacturers for tracking solar UV exposure behavior at high-sample rates by employing cost effective infrared photodiode technology. Future implementations of an infrared ESJ for monitoring personal sun exposure behavior could be built into weather resistant (or submersible) housings, provided the infrared photodiode sensor remains unobstructed and exposed to the environment.

When placed behind glass, or the windows of a building an infrared ESJ will record positive exposure readings. Glass is an effective absorber of UVB radiation, but will generally transmit UVA and near infrared wavelengths in the absence of varied glazing techniques or protective tinting. Thus, the ESJ would record personal exposures near large window frames or greenhouses, for example. This is a limitation to be considered should study participants be regularly working behind glass when studying biologically effective exposure patterns sensitive to short UVB wavelengths, including erythema (29), vitamin D (39), and some conditions of the eye such as photokeratitis (40). Future trends in window glass, including electrochromatic windows which can act as effective absorbers of near infrared wavelengths (41), and small window glass frames offering low sky views will, however, likely result in indoor exposures being correctly monitored by a cost effective infrared ESJ. The circuit as designed has some further limitations which require either the use of self-reported sun diaries or electronic apps depending on particular study designs for monitoring personal sun exposure behavior. The ESJ does not monitor the level of personal protection applied on a day to day basis. This includes monitoring of personal sun screen use, clothing, and hat types worn while outdoors. The ESJ does not record phenotype characteristics, age or gender information of study participants. For many studies, this will need to be recorded in addition to personal exposure pattern behavior.

The developed ESJ avoids uncertainties associated with participants recalling from memory periods of daily outdoor exposure by monitoring second-long exposure periods (extending up to $4 \frac{1}{2} \mathrm{~h}$ using the TK-4703 data logger). The data logger used with the current version of the ESJ can be set to log signal voltages over variable time periods as desired, extending the range over which the
ESJ can be used. The circuit as presented in Figure 1 could also be attached to any other readily available data logger. This may include, for example, smart phone memory or data loggers similar to those found in commercially available fitness trackers. The length of time the ESJ can record depends on the memory capacity of the logger used. An 8-bit (1 byte) digital logger can store up to 256 distinct voltage levels in the $0-2.5 \mathrm{~V}$ range for each byte of available memory. Thus, 1 megabyte of free memory could store up to 1,048,576 million data samples. At a sample resolution of $1 \mathrm{~s}$, even this moderate memory capacity would allow storage over 12 continuous days. As designed, the ESJ draws as very low current from the $3 \mathrm{~V}$ battery source. All tests performed in this research, including preliminary development were completed using a single battery exceeding $20 \mathrm{~h}$ continuous operation. We estimate the ESJ, configured using the components presented in Table 2 could run continuously for several weeks without the need for a battery change.

An infrared photodiode, employed on a wrist band, lapel, or vertex of a cap would provide a significant amount of data on personal sunlight exposure patterns that can be monitored without the requirement of employing typically more expensive UV photodiodes (Table 1). A cost effective infrared ESJ has the potential to greatly extend study sample sizes. That infrared diodes are readily available, and already often deployed on devices such as smartphone cameras for sensing ambient light conditions demonstrates their potential to be employed for monitoring personal outdoor exposure patterns en masse. An infrared ESJ provides an opportunity to collect more extensive data sets from large population groups that may prove valuable in linking outdoor behavior patterns with causes of sun-related disease.

\section{AUTHOR CONTRIBUTIONS}

ND drafted the original manuscript, including Sections "Introduction," "MaterialsandMethods," "Results," and "Discussion." AP made original contributions to the draft manuscript, providing editing assistance, and modifications to the original study design now presented in Section "Materials and Methods" of the article. $\mathrm{HB}$ made a significant editing contribution to the work, including rewriting of Sections "Materials and Methods," "Results," and "Discussion" of the manuscript. AR developed the figures presented in the article, collected research data, and designed the algorithms used to extract exposure information presented in the manuscript results. AR contributed to the final editing of the manuscript. RE played a key role in the original study design. RE collected some of the research data presented in the manuscript results and made original contributions to Sections "Introduction" and "Discussion."

\section{ACKNOWLEDGMENTS}

The authors would like to acknowledge the support of the Faculty of Health, Engineering and Sciences, University of Southern Queensland.

\section{FUNDING}

This research did not receive any specific grant from funding agencies in the public, commercial, or not-for-profit sectors. 


\section{REFERENCES}

1. International Agency for Research on Cancer. IARC Monographs on the Evaluation of Carcinogenic Risks to Humans, Solar and Ultraviolet Radiation. (Vol. 100D). (2012). p. 35-101. Available from: http://monographs.iarc.fr/ ENG/Monographs/vol100D/index.php

2. Kim Y, He YY. Ultraviolet radiation-induced non-melanoma skin cancer: regulation of DNA damage repair and inflammation. Genes Dis (2014) 1:188-98. doi:10.1016/j.gendis.2014.08.005

3. Leiter U, Eigentler T, Garbe C. Epidemiology of skin cancer. In: Sunlight, Vitamin D and Skin Cancer. New York, NY: Springer (2014). p. 120-40.

4. Yam JCS, Kowok AKH. Ultraviolet light and ocular diseases. Int Ophthalmol (2014) 34:383-400. doi:10.1007/s10792-013-9791-x

5. Jhappan C, Noonan FP, Merlino G. Ultraviolet radiation and cutaneous malignant melanoma. Oncogene (2013) 22:3099-112. doi:10.1038/sj.onc.1206450

6. Thiel S, Stiener K, Seidlitz HK. Modification of erythemally effective irradiance by clouds. Photochem Photobiol (1997) 65:969-73. doi:10.111 1/j.1751-1097.1997.tb07956.x

7. Kerr JB, Fioletov VE. Surface ultraviolet radiation. Atmos Ocean (2008) 46:159-84. doi:10.3137/ao.460108

8. Bais AF, McKenzie RL, Bernhard G, Aucamp PJ, Ilyas M, Madronich S, et al. Ozone depletion and climate change: impacts on UV radiation. Photochem Photobiol Sci (2015) 14:19-52. doi:10.1039/c4pp90032d

9. Diffey BL, Saunders PJ. Behaviour outdoors and its effects on personal ultraviolet exposure rate measured using and ambulatory datalogging dosimeter. Photochem Photobiol (1995) 61:615-8. doi:10.1111/j.1751-1097.1995. tb09877.x

10. Coups EJ, Manne SL, Heckman CJ. Multiple skin cancer risk behaviors in the U.S. population. Am J Prev Med (2008) 34:87-93. doi:10.1016/j. amepre.2007.09.032

11. Diffey BL, Gibson CJ, Haylock R, McKinlay AF. Outdoor ultraviolet exposure of children and adolescents. Br J Dermatol (1996) 134:1030-4. doi:10.111 1/j.1365-2133.1996.tb07937.x

12. Moehrle M. Outdoor sports and skin cancer. Clin Dermatol (2008) 26:12-5. doi:10.1016/j.clindermatol.2007.10.001

13. Downs NJ, Parisi AV, Igoe D. Measurements of occupational ultraviolet exposure and the implications of timetabled yard duty for school teachers in Queensland, Australia: preliminary results. J Photochem Photobiol B (2014) 131:84-9. doi:10.1016/j.jphotobiol.2014.01.012

14. Downs NJ, Harrison SL, Garzon-Chavez DR, Parisi AV. Solar ultraviolet and the occupational radiant exposure of Queensland school teachers: a comparative study between teaching classifications and behavior patterns.J Photochem Photobiol B (2016) 158:105-12. doi:10.1016/j.jphotobiol.2016.02.018

15. Harrison SL, Garzon-Chavez DR, Nikles CJ. Sun protection policies of Australian primary schools in a region of high sun exposure. Health Educ Res (2016) 31:416-28. doi:10.1093/her/cyw020

16. Davis A, Deane GHW, Diffey BL. Possible dosimeter for ultraviolet radiation. Nature (1976) 261:169-70. doi:10.1038/261169a0

17. Moehrle M, Garbe C. Personal UV dosimetry by Bacillus subtilis spore films. Dermatology (2000) 200:1-5. doi:10.1159/000018306

18. O’Riordan DL, Stanton WR, Eyeson-Annan M, Gies P, Roy C. Correlations between reported and measured ultraviolet radiation exposure of mothers and young children. Photochem Photobiol (2000) 71:60-4. doi:10.1562/ 0031-8655(2000)071<0060:CBRAMU>2.0.CO;2

19. Schouten P, Parisi AV, Turnbull DJ. Evaluation of a high exposure solar UV dosimeter for underwater use. Photochem Photobiol (2007) 83:931-7. doi:10.1111/j.1751-1097.2007.00085.x

20. Idorn LW, Datta P, Heydenreich J, Philipsen PA, Wulf HC. Sun behavior after cutaneous malignant melanoma: a study based on ultraviolet radiation measurements and sun diary data. Br J Dermatol (2013) 168:367-73. doi:10.1111/ bjd. 12066

21. Dobbinson S, Niven P, Buller D, Allen M, Gies P, Warne C. Comparing handheld meters and electronic dosimeters for measuring ultraviolet levels under shade and in the sun. Photochem Photobiol (2016) 92:208-14. doi:10.1111/ php. 12551

22. Thieden E, Philipsen PA, Wulf HC. Ultraviolet radiation exposure pattern in wintercompared with summerbased on time-stampedpersonaldosimeterreadings. Br J Dermatol (2006) 154:133-8. doi:10.1111/j.1365-2133.2005.06961.x
23. El Naggar S, Gustat H, Magister H, Rochlitzer R. An electronic UVB-dosimeter. J Photochem Photobiol B (1995) 31:83-6. doi:10.1016/1011-1344 (95)07216-2

24. Bodekaer M, Harrison GI, Philipsen P, Petersen B, Triguero-Mas M, Schmalwieser AW, et al. Personal UVR exposure of farming families in four European countries. J Photochem Photobiol B (2015) 153:267-75. doi:10.1016/j. jphotobiol.2015.10.002

25. Muñoz E, Monroy E, Calle F, Omnès F, Gibart P. AlGaN photodiodes for monitoring solar UV radiation. J Geophys Res (2000) 105:4865-71. doi:10.1029/ 1999JD900939

26. Allen MW, McKenzie RL. Electronic UV dosimeters for research and education. NIWA UV Workshop. Queenstown, New Zealand (2010). p. 7-9.

27. McKenzie R, Liley B, Allen M, Sherman Z. Measuring personal UV exposure with electronic dosimeters. The 3rd International Conference on UV and Skin Cancer Prevention. Melbourne (2015).

28. Wulf HC, Gniadecka M. Electronic UV dosimeters. Skin Res Technol (1996) 2:103-7. doi:10.1111/j.1600-0846.1996.tb00070.x

29. Commission Internationale de l'Eclairage (CIE). Erythema Reference Action Spectrum and Standard Erythema Dose. Vienna: CIE (1998). ISO 17166:1999 (CIE S 007/E:1998).

30. Diffey BL, Jansén CT, Urbach F, Wulf HC. The standard erythema dose: a new photobiological concept. Photodermatol Photoimmunol Photomed (1997) 13:64-6. doi:10.1111/j.1600-0781.1997.tb00110.x

31. Vanicek K, Frei T, Lytinska Z, Schmalwieser A. UV-Index for the Public: a Guide for Publication and Interpretation of Solar UV Index Forecasts for the Public Prepared by the Working Group 4 of the COST-713 Action 'UVB Forecasting'. Brussels, Belgium: Publication of the European Communities (1999).

32. Cheuk M, Xu D, McLean R. Delivery of personal ultraviolet radiation information to smartphones. NIWA UV Workshop. Auckland, New Zealand (2014). Available from: https://www.niwa.co.nz/atmosphere/uv-ozone/ uv-science-workshops/2014-uv-workshop

33. Turner J, Parisi AV, Downs N, Lynch M. From ultraviolet to Prussian blue: a spectral response for the cyanotype process and a safe educational activity to explain UV exposure for all ages. Photochem Photobiol Sci (2014) 13:1753-64. doi:10.1039/C4PP00166D

34. Liu X, Vega K, Maes P, Paradiso JA. Wearability factors for skin surfaces. Proceedings of the 7th Augmented Human International Conference. Geneva, Switzerland (2016). Available from: http://resenv.media.mit.edu/pubs/papers/ skin_interface.pdf

35. Steve Spangler Science. UV Color Changing Beads. (2017). Available from: https://www.stevespanglerscience.com/store/uv-color-changing-beads.html

36. Solar Active. UV Awareness Bracelets. (2017). Available from: https://www. solaractive.com/wholesale-promotional/uv-awareness-bracelets/

37. Vishay Semiconductors. BPV22F Product Information. (2017). Available from: https://www.vishay.com/product?docid $=81508$

38. Gemini Data Loggers. Tinytag Talk 2: TK-4703-PK. (2017). Available from: http://www.geminidataloggers.com/data-loggers/tinytag-talk-2/tk-4703

39. Bouillon R, Eisman J, Garabedian M, Holick M, Klienschmidt J, Suda T, et al. Action Spectrum for the Production of previtamin $D_{3}$ in Human Skin. Technical Report 174. Vienna, Austria: Commission Internationale de l'Eclairage (CIE) (2006). Available from: http://div6.cie.co.at/?i_ca_ $\mathrm{id}=611$ \&pubid $=187$

40. Commission Internationale de l'Eclairage (CIE). Photokeratitis. Research Note CIE J 5. Vienna: CIE (1986). p. 19-23.

41. Jelle BP. Solar radiation glazing factors for window panes, glass structures and electrochromatic windows in buildings: measurement and calculation. Sol Energ Mat Sol C (2013) 116:291-323. doi:10.1016/j.solmat.2013.04.032

Conflict of Interest Statement: The authors declare that the research was conducted in the absence of any commercial or financial relationships that could be construed as a potential conflict of interest.

Copyright (c) 2017 Downs, Parisi, Butler, Rawlings and Elrahoumi. This is an open-access article distributed under the terms of the Creative Commons Attribution License (CC BY). The use, distribution or reproduction in other forums is permitted, provided the original author(s) or licensor are credited and that the original publication in this journal is cited, in accordance with accepted academic practice. No use, distribution or reproduction is permitted which does not comply with these terms. 